\title{
Replacement of butter on bread by rapeseed oil and rapeseed oil- containing margarine: effects on plasma fatty acid composition and serum cholesterol
}

\author{
BY T. SEPPÄNEN-LAAKSO ${ }^{1}$, H. VANHANEN ${ }^{2}$, I. LAAKSO ${ }^{1}$, H. KOHTAMÄKI \\ AND J. VIIKARI ${ }^{3}$ \\ ${ }^{1}$ Department of Pharmacy, Fabianinkatu 35, SF-00170 Helsinki and ${ }^{2}$ Second Department of \\ Medicine, University of Helsinki, Finland \\ ${ }^{3}$ Department of Medicine, University of Turku, Finland
}

(Received 18 February 1991-Accepted 29 October 1991)

\begin{abstract}
The effects of zero-erucic acid rapeseed oil and rapeseed oil-containing margarine on plasma fatty acid composition and serum cholesterol were studied in butter users $(n$ 43). Compliance to the substitution was followed by fatty acid analysis of total plasma and plasma phospholipids. The amount of substitute fats represented, on average, $21 \%$ of total fat and $8 \%$ of total energy intake. Changes in the relative fatty acid composition of plasma phospholipids indicated further fatty acid metabolism, and were closely related to the serum cholesterol level. The reduction in saturated fatty acids led to a significant increase in the proportion of $n-3$ and $n-6$ polyunsaturated fatty acids (PUFA) with the rapeseed oil diet, whereas the margarine caused a significant rise in n-6 PUFA only. The increase in the proportions of the two PUFA families occurred in accordance with their competitive order, most completely with the rapeseed oil diet. When butter was replaced by rapeseed oil, low-density-lipoprotein-cholesterol decreased by an average of $9.1 \%$ without a reduction in high-density-lipoprotein-cholesterol. During margarine substitution the reduction was $5.2 \%$, on average. Of the plasma phospholipids, $\alpha$-linolenic acid and the linoleic:stearic acid ratio, but not oleic acid, were the components most significantly correlated with serum cholesterol levels or the decrease in these levels. The results show that rapeseed oil can act primarily as a source of essential fatty acids, rather than that of monoenes, in the diet of butter users.
\end{abstract}

Zero-erucic acid rapeseed oil: Plasma fatty acids: $\alpha$-Linolenic acid: Serum cholesterol

Low-density-lipoprotein-cholesterol (LDL-C) constitutes a major risk factor for coronary heart disease (Keys, 1970). Saturated fat has been clearly demonstrated to raise both total cholesterol (TC) and LDL-C levels (Hegestedt et al. 1965), in contrast to hypocholesterolaemic polyunsaturated fatty acids (PUFA; Nestel, 1987). Linoleic acid (LA) plays an important role, since a low proportion in plasma and adipose tissue lipids is considered to be associated with a high incidence of coronary heart disease (Logan et al. 1978; Riemersma et al. 1986). Dietary modification is the first step in lowering cholesterol levels. It is recommended that the intake of saturated fat be restricted, and the use of vegetable oils containing mainly monounsaturated fatty acids (MUFA) and PUFA be favoured.

Zero-erucic acid turnip rape (Brassica campestris) varieties, cultivated on an extensive scale during the last decade, have become an important source of vegetable oil also in Finland (Laakso, 1986). Rapeseed oil (canola-type oil), owing to its versatile fatty acid composition consisting of essential linoleic (about 22\%) and $\alpha$-linolenic acids ( $\alpha$-LLA; about $11 \%$ ) and high content of MUFA (about $60 \%$ ), can be used to balance a high- 
saturated-fat diet. Rapeseed oil also has the lowest saturated fatty acid (SaFA) content of major vegetable oils (about 6\%; Ackman, 1983).

A reduction in cholesterol levels during rapeseed oil substitution has been demonstrated in several studies (Savoie et al. 1983; Renaud et al. 1986; McDonald et al. 1989). The cholesterol-lowering effect of rapeseed oil has been attributed to its high content of oleic acid (OA), whereas PUFA are responsible for the anti-thrombotic effects (McDonald et al. 1989). $\alpha$-LLA acts as a precursor of eicosapentaenoic acid (EPA) in man (Sanders \& Younger, 1981), and an increase in plasma EPA levels has been reported during rapeseed oil supplementation (Lassere \& Jacotot, 1983; Renaud et al. 1986; Weaver et al. 1990).

In studies dealing with cholesterol the dietary control is usually based on dietary diaries and evaluation of nutrient intake. The decrease in cholesterol levels is often attributed to the most prominent change made in the composition of dietary fat, without measuring the response at the plasma fatty acid level. Thus, the possible relationships between minor compounds and cholesterol have been largely ignored. Furthermore, when experiments are started with a high intake of SaFA the determination of which fatty acids are primarily needed in the diet may be obscured. The fatty acid composition of total plasma reflects the dietary fat within a couple of days, whereas that of phospholipids is more stable and representative of the type of dietary fat consumed during a longer period of time (Dougherty et al. 1987; Moilanen, 1987). Determination of the plasma phospholipid-fatty acid pattern is also important, since a low PUFA and high SaFA concentration are considered to be a predictor of coronary heart diseases (Miettinen et al. 1982).

The aim of the present study was to examine the effects of partial rapeseed oil and margarine substitution on plasma fatty acid composition and serum cholesterol in butter users. The only change made to the diet was the replacement of butter on bread by rapeseed oil or rapeseed-oil-containing margarine. Relationships between plasma fatty acids and serum cholesterol were also studied.

\section{SUBJECTS AND METHODS}

\section{Subjects}

The screening study was carried out in the Turku area (SW Finland) in January 1988 (Fig. 1). A total of 400 persons of working age were screened, and those with serum TC levels between 5.0 and $8.5 \mathrm{mmol} / 1$ and triacylglycerols (TG) less than $3.5 \mathrm{mmol} / 1$ were accepted for further investigation. The majority of the subjects were excluded owing to minor use of bread and butter $(<3$ slices of bread/d) or drug therapy, and the use of fat-containing nutritional supplements such as cod-liver oil or evening primrose (Oenothera biennis) seed oil.

The final grouping was done according to the baseline samples taken at the end of February. Subjects who used butter on bread were divided into rapeseed oil $(n 20)$ and margarine ( $n$ 23) groups alphabetically. A control group ( $n$ 11), which consisted of persons who used both butter and common LA-rich margarines on bread (mixed fat users, Fig. 1), was used to monitor changes in lipid levels during the trial period from February to May. The substitution groups were similar as regards gender and age, whereas the mean age and body mass index (BMI; $\mathrm{kg} / \mathrm{m}^{2}$ ) were slightly lower (not significant; NS) in the final control group (Table 1).

\section{Substitute fats}

Rapeseed oil was used on bread as a water-oil emulsion (fat content $650 \mathrm{~g} / \mathrm{kg}$ ). The margarine contained rapeseed $(240 \mathrm{~g} / \mathrm{kg})$, sunflower, coconut and partially hydrogenated soyabean oils (fat content $800 \mathrm{~g} / \mathrm{kg}$ ). 


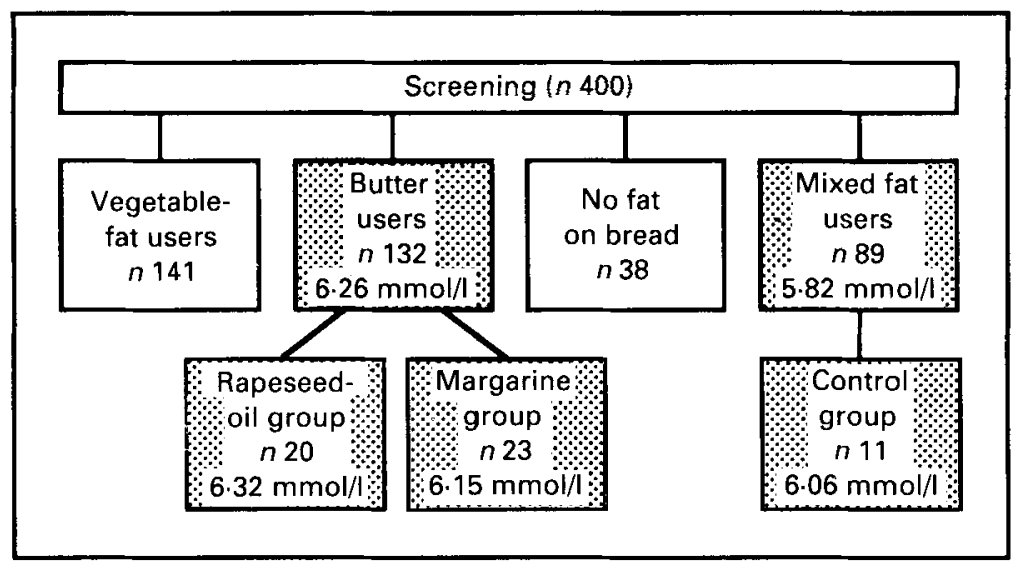

Fig. I. Screening and grouping of the subjects. (圆), Experimental groups for whom the mean total cholesterol values are shown

Table 1. Characteristics of the dietary groups

\begin{tabular}{lrrrrrr} 
& \multicolumn{3}{c}{ Subjects } & & \\
\cline { 2 - 5 } Group* & $n$ & Men & Women & $\begin{array}{c}\text { Age } \\
\text { (years) }\end{array}$ & BMI \\
\hline Rapeseed oil & 20 & 10 & 10 & $45 \cdot 7$ & $25 \cdot 7$ \\
Margarine & 23 & 11 & 12 & $44 \cdot 8$ & $25 \cdot 4$ \\
Control & 11 & 5 & 6 & $41 \cdot 0$ & $24 \cdot 7$ \\
\hline
\end{tabular}

BMI, body mass index $\left(\mathrm{kg} / \mathrm{m}^{2}\right)$.

* For details of screening, see Fig. 1.

The substitution period during which the butter on bread was replaced by these fats lasted for 6 weeks. No other changes were made in the diet. During the post-experimental period ( 6 weeks) the subjects returned to using butter on their bread. The control group was asked not to make any dietary changes during the 12-week trial.

The fatty acid composition of the substitute fats, butter and rapeseed oil, as determined by gas-liquid chromatography (GLC; Seppänen-Laakso et al. 1990), are presented in Table 2.

\section{Dietary control}

All the subjects ( $n$ 54) kept 3 d dietary diaries at the baseline and at 6 weeks, and the data obtained were analysed for nutrients using a computerized food table-based Nutrica programme (Table 3; Seppänen, 1987). The consumption of substitute fats given in portions was controlled twice (at 3 and 6 weeks). Body-weight and BMI were recorded at each blood sample withdrawal.

\section{Blood samples and lipid analyses}

Fasting blood samples were taken at the baseline, at 3 and 6 weeks of substitution, and at the end of the post-experimental period. Compliance to the substitution was followed by total plasma and plasma phospholipid fatty acid analyses using optimized capillary GLC techniques. The reproducibility of the method expressed as the mean relative standard 
Table 2. Fatty acid composition ( $\mathrm{g} / 100 \mathrm{~g}$ total fatty acids) of substitute fats, butter and rapeseed oil

\begin{tabular}{|c|c|c|c|c|c|}
\hline Fatty acids & & $\begin{array}{l}\text { Rapeseed oil } \\
\text { emulsion* }\end{array}$ & Margarine $\dagger$ & Butter $\ddagger$ & $\begin{array}{l}\text { Zero-erucic acid } \\
\text { rapeseed oil } \neq\end{array}$ \\
\hline \multicolumn{6}{|l|}{ Saturated } \\
\hline $10: 0-14: 0$ & & 0.1 & $4 \cdot 5$ & $19 \cdot 7$ & 0.1 \\
\hline Palmitic acid & $(16: 0)$ & $3 \cdot 3$ & $8 \cdot 0$ & $36 \cdot 0$ & $3 \cdot 3$ \\
\hline Stearic acid & $(18: 0)$ & 1.5 & $7 \cdot 2$ & $13 \cdot 8$ & 1.5 \\
\hline $20: 0-24: 0$ & & 0.8 & 0.9 & $0 \cdot 2$ & 0.7 \\
\hline Total & & $5 \cdot 7$ & $20 \cdot 6$ & $69 \cdot 7$ & $5 \cdot 6$ \\
\hline \multicolumn{6}{|l|}{ Monounsaturated } \\
\hline Palmitoleic acid & $(16: 1 n-7)$ & $0 \cdot 1$ & $0 \cdot 1$ & 1.6 & 0.2 \\
\hline \multirow[t]{2}{*}{ Oleic acid } & $(18: 1 c i s)$ & $57 \cdot 2$ & $31-1$ & $24 \cdot 3$ & $57 \cdot 0$ \\
\hline & (18:1 trans) & - & $16 \cdot 4$ & 1.9 & - \\
\hline Eicosenoic acid & $(20: \ln -9)$ & 1.6 & 0.6 & $0 \cdot 3$ & 1.5 \\
\hline Erucic acid & $(22: \ln -9)$ & 0.9 & $0 \cdot 3$ & - & 0.9 \\
\hline Total & & $59 \cdot 8$ & $48 \cdot 5$ & $28 \cdot 1$ & $59 \cdot 6$ \\
\hline \multicolumn{6}{|l|}{ Polyunsaturated } \\
\hline Linoleic acid & $(18: 2 n-6)$ & $24 \cdot 3$ & $28 \cdot 1$ & $1 \cdot 7$ & $23 \cdot 5$ \\
\hline$\alpha$-Linolenic acid & $(18: 3 n-3)$ & $10 \cdot 1$ & $2 \cdot 7$ & 0.5 & $11 \cdot 2$ \\
\hline Eicosadienoic acid & $(20: 2 n-6)$ & 0.1 & $0 \cdot 1$ & - & $0 \cdot 1$ \\
\hline Total & & $34 \cdot 5$ & $30 \cdot 9$ & $2 \cdot 2$ & $34 \cdot 8$ \\
\hline
\end{tabular}

deviation (RSD) was 2.4 and $3.9 \%$ for fourteen total plasma and twelve plasma phospholipid fatty acids respectively (Seppänen-Laakso et al. 1990). The percentage of the methodological variation ('error') relative to the total variation ranged from 0.5 to 6.4 for fourteen total plasma fatty acids of 100 subjects. The corresponding percentages for $\alpha-$ LLA, LA, OA and EPA in plasma phospholipids were $3.2,0.9,1.0$ and 1.7 respectively, whereas in the case of palmitic and stearic acid the methodological variation represented 18 and $9 \%$ of the total variation respectively (Seppänen-Laakso et al. 1990).

Serum TC and TG values were determined enzymically, as well as high-densitylipoprotein-cholesterol (HDL-C), after precipitation with polyethyleneglycol (PEG-6000; final concentration $100 \mathrm{~g} / 1$; Viikari, 1976). LDL-C levels were calculated according to the Friedewald et al. (1972) formula.

Statistical differences in lipid levels were analysed by the paired $t$ test. Pearson's correlation coefficients and regression were used to study the relationships between fatty acid composition and cholesterol concentration. All the significance levels are given twosided.

\section{RESULTS \\ Dietary characteristics}

The consumption of substitute fats given in portions varied from 8 to $46 \mathrm{~g} / \mathrm{d}$ (as $100 \%$ fat), the mean dose being $18 \mathrm{~g} / \mathrm{d}$ in the rapeseed-oil group and $23 \mathrm{~g} / \mathrm{d}$ in the margarine group. According to the dietary diaries (Table 3) substitute fat accounted for 20 and $22 \%$ of the total fat intake in the rapeseed-oil and margarine groups, corresponding to 7 and $8 \%$ of the total energy intake respectively. No significant changes in fat intake were registered during the study and body-weights remained unchanged. 
Table 3. Mean daily nutrient intakes of groups of subjects $\dagger$ substituting butter on bread by rapeseed oil or rapeseed oil-containing margarine $\$$

\begin{tabular}{|c|c|c|c|c|c|c|}
\hline \multirow[b]{2}{*}{ Nutrient§ } & & \multicolumn{2}{|c|}{ Rapeseed-oil group } & \multicolumn{2}{|c|}{ Margarine group } & \multirow{2}{*}{$\frac{\text { Control group }}{\text { Mean }^{\|}}$} \\
\hline & & $\begin{array}{c}\text { Before } \\
\text { substitution }\end{array}$ & $\begin{array}{c}\text { During } \\
\text { substitution }\end{array}$ & $\begin{array}{c}\text { Before } \\
\text { substitution }\end{array}$ & $\begin{array}{c}\text { During } \\
\text { substitution }\end{array}$ & \\
\hline \multirow{2}{*}{ Energy intake: } & $(\mathrm{kJ})$ & 9226 & 8597 & 8533 & 8451 & 8352 \\
\hline & (kcal) & 2200 & 2050 & 2030 & 2010 & 1990 \\
\hline Protein & & 15.6 & $12 \cdot 9 * \downarrow$ & $15 \cdot 5$ & $15 \cdot 9$ & $15 \cdot 1$ \\
\hline \multicolumn{7}{|l|}{ Fats: } \\
\hline Total & & 38.8 & $40 \cdot 4$ & 37.6 & 37.9 & $36 \cdot 4$ \\
\hline SaFA & & 17.4 & $14 \cdot 0^{* *} \downarrow$ & $16 \cdot 8$ & $15 \cdot 5$ & $15 \cdot 5$ \\
\hline MUFA & & $12 \cdot 2$ & $15 \cdot 1 * \uparrow$ & $12 \cdot 0$ & $12 \cdot 8$ & $13 \cdot 2$ \\
\hline PUFA & & 5.6 & $8 \cdot 1^{* * \uparrow}$ & $5 \cdot 3$ & $6 \cdot 5^{* \uparrow}$ & $5 \cdot 5$ \\
\hline$P: S$ & & 0.34 & $0.67 * * \uparrow$ & $0 \cdot 33$ & $0 \cdot 46^{* * \uparrow} \uparrow$ & 0.40 \\
\hline Fat on bread: & & $6 \cdot 2$ & 6.8 & $7 \cdot 3$ & 7.9 & 9.7 \\
\hline & (g/kg fat) & 167 & 202 & 194 & 219 & 264 \\
\hline Carbohydrates & & $39 \cdot 4$ & $40 \cdot 0$ & $40 \cdot 9$ & $41 \cdot 0$ & $39 \cdot 6$ \\
\hline Alcohol & & $1 \cdot 1$ & 1.6 & $1 \cdot 3$ & $0 \cdot 4$ & $3 \cdot 3$ \\
\hline Cholesterol & $(\mathrm{mg} / \mathrm{d})$ & 377 & 293 & 436 & $319^{*} \downarrow$ & 345 \\
\hline Fibre, gel-forming & $(g / d)$ & 22.9 & $24 \cdot 7$ & $19 \cdot 0$ & $19 \cdot 3$ & $19 \cdot 4$ \\
\hline
\end{tabular}

SaFA, saturated fatty acids; MUFA, monounsaturated fatty acids; PUFA, polyunsaturated fatty acids; P:S, PUFA:SaFA; $\uparrow$, increase; $\downarrow$, decrease.

Mean values were significantly different from corresponding baseline values (paired $t$ test). ${ }^{*} P<0.05$, ** $P<0.01$.

$\dagger$ For details of screening procedures and groups of subjects, see Table 1 and Fig. 1.

$\ddagger$ For details of fatty acid composition, see Table 2 .

$\$ \%$ of total energy unless otherwise indicated.

Mean of $6 \mathrm{~d}$.

The PUFA:SaFA ratio (P:S) of dietary fat doubled during rapeseed oil substitution, since the proportion of SaFA decreased and that of PUFA increased significantly (Table 3). The proportion of MUFA also increased. During margarine substitution the rise in $P: S$ ratio was primarily due to an increase in PUFA. No significant changes were found in daily nutrient intake in the control group and, therefore, the data in Table 3 are expressed as the mean values of two $3 \mathrm{~d}$ dietary diaries.

The average intake of $\alpha$-LLA derived from rapeseed oil supplementation was $1.8 \mathrm{~g} / \mathrm{d}$, covering approximately $20 \mathrm{~g} / \mathrm{kg}$ total fat $(0.8 \%$ of total energy). The mean amount of trans fatty acids derived from margarine was $3.7 \mathrm{~g} / \mathrm{d}$, covering about $43 \mathrm{~g} / \mathrm{kg}$ total fat $(1.7 \%$ of total energy).

\section{Total plasma fatty acids}

The most characteristic constituents in the substitute fats were OA, $(18: 1 n-9)$ and LA (18:2n-6) and additionally $\alpha$-LLA $(18: 3 n-3)$ in rapeseed oil (Table 2$)$. The changes in their proportions in total plasma, as well as those of total SaFA and EPA $(20: 5 n-3)$, are presented in Table 4.

The proportion of $\alpha$-LLA increased during the rapeseed-oil diet $(P<0.001)$, and decreased to the baseline level at the end of the study (Table 4). The rise correlated with the amount of rapeseed oil used $(r+0 \cdot 51, P<0.05, n 20)$. The most significant increase in the relative amount of LA occurred at 6 weeks in both substitution groups $(P<0 \cdot 001$; Table 4). During the 6 week post-experimental period the values did not completely revert to the baseline level. Changes in the plasma OA levels were slight and resembled those of 


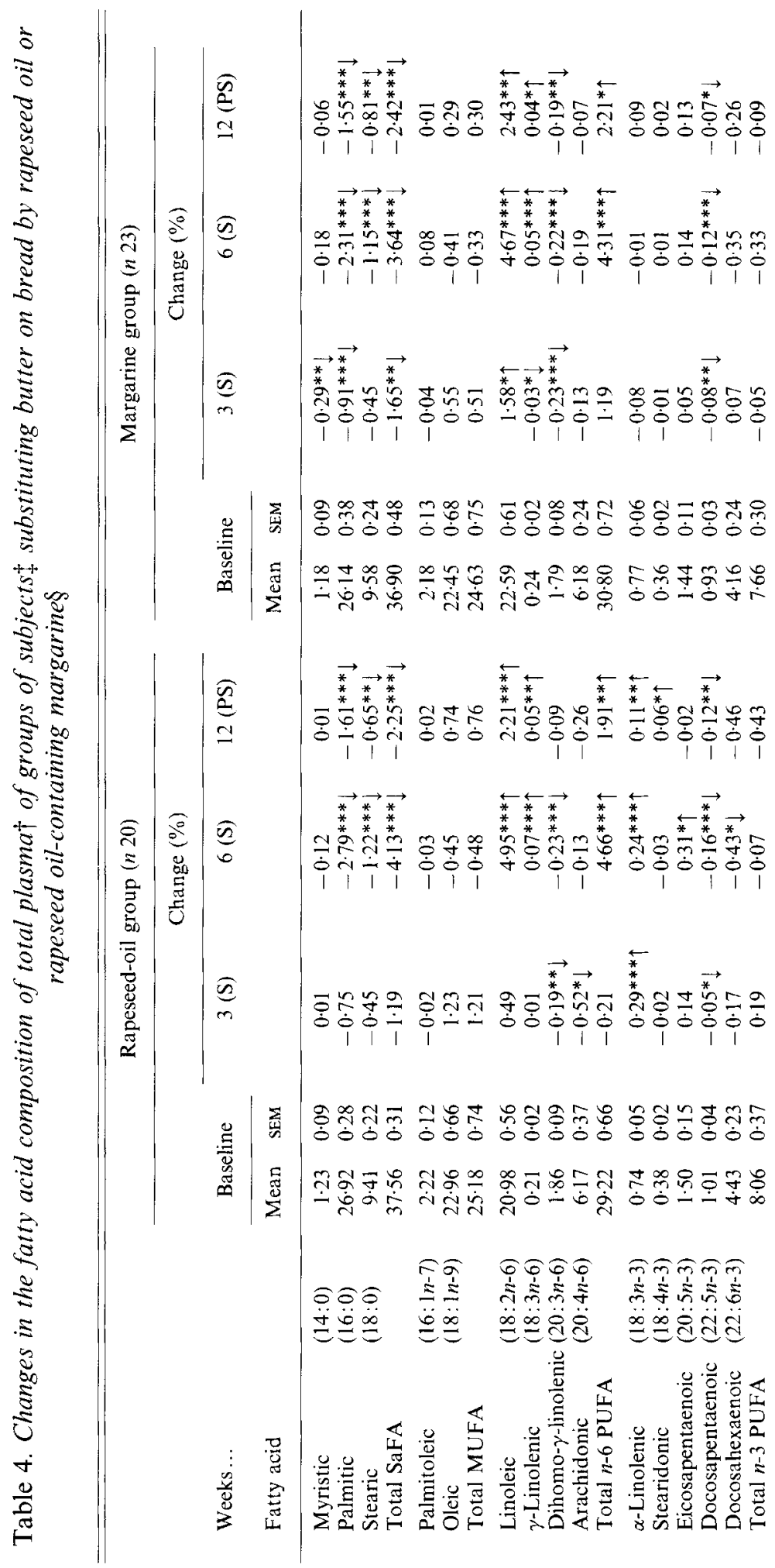




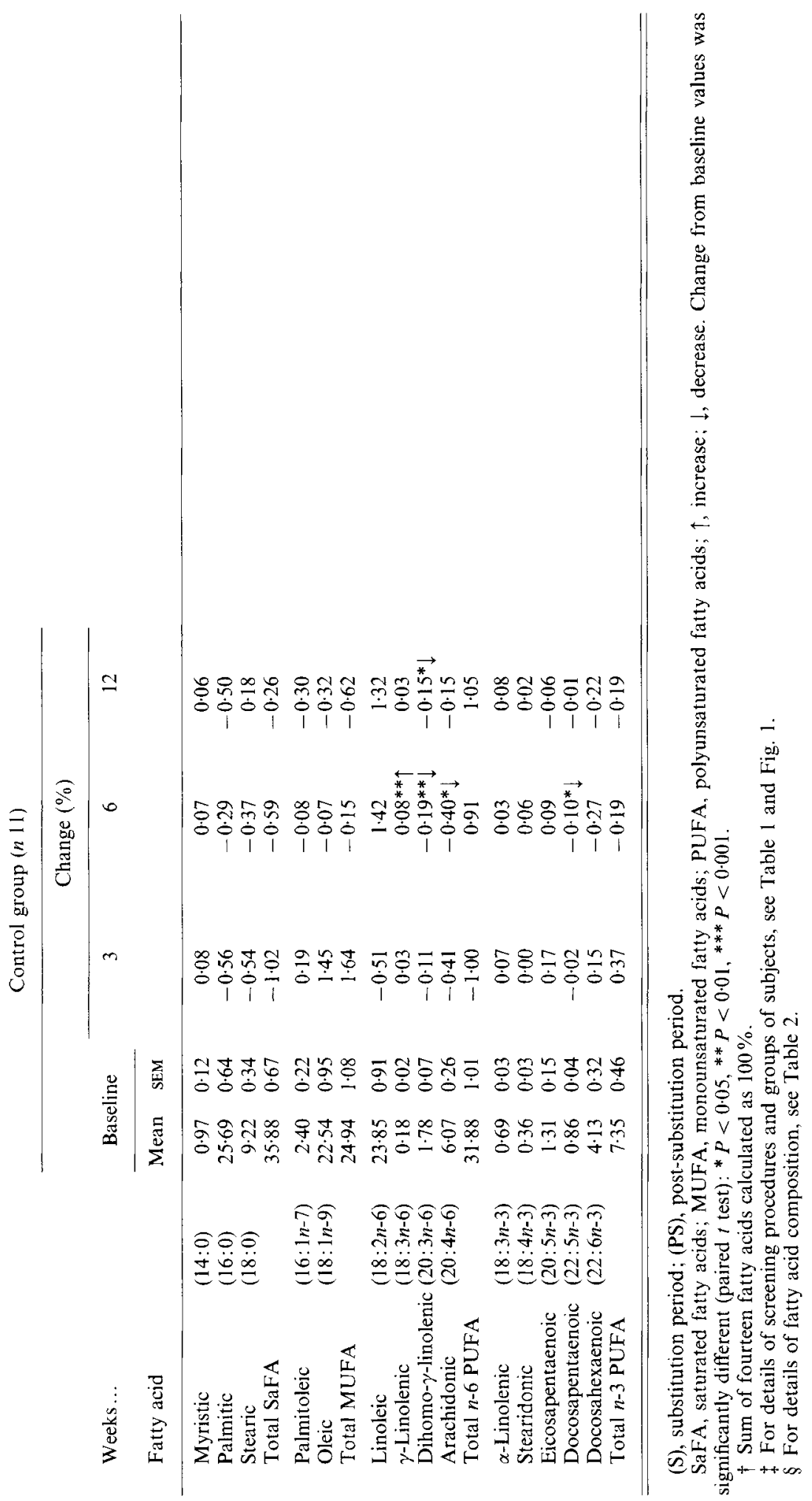


the control group. The proportion of SaFA was lowest after the 6 week substitution period and did not reach the baseline level at 12 weeks, although the subjects had returned to using butter during the post-experimental period. The changes in the control group as regards the main dietary fatty acids were clearly minor and not significant (Table 4).

\section{Plasma phospholipid fatty acids}

The changes in the fatty acid composition of the plasma phospholipids are presented in Table 5. The proportion of $\alpha$-LLA increased considerably in the rapeseed-oil group at 3 weeks $(P<0.001)$, but returned to the baseline level at 6 weeks. In both substitution groups the increase in the relative amount of LA was clearly less than that in total plasma. In the case of OA a significant decrease was found at 6 weeks in the rapeseed-oil group. The proportion of SaFA fell during the first 3 weeks, but at 6 weeks a partial restoration occurred in contrast to total plasma. At 6 weeks the relative amount of stearic acid returned to the baseline level in both substitution groups (Table 5).

The proportion of $n-3$ PUFA including EPA increased in the rapeseed-oil group after 3 weeks substitution $(1.7 \%, P<0.001$ ), and remained unchanged for the next 3 weeks (Table 5). The increase in $n-3$ PUFA was greater than that in the margarine group at both 3 $(P<0.05)$ and 6 weeks $(P<0.01$, unpaired $t$ test $)$. A significant rise in the level of $n-6$ PUFA did not occur until the end of substitution, when there was a simultaneous decrease in MUFA $(-2.3 \%, P<0.001)$. The proportion of SaFA fell after 3 weeks substitution $(-2.9 \%, P<0.001)$, remaining below the baseline value at the end of the experiment $(P<0.01)$. This reduction at 3 weeks was more pronounced $(P<0.05)$ than that during margarine substitution.

In the margarine group the decrease in the relative amount of SaFA led to an increase in the proportion of $n-6$ PUFA already during the first 3 weeks $(P<0 \cdot 01$; Table 5). Most of the significant changes in the control group occurred between SaFA and MUFA during the post-experimental period.

\section{Serum lipids}

In the rapeseed-oil group the serum TC and LDL-C decreased by $0.49 \mathrm{mmol} / 1(7.8 \%)$ and $0.59 \mathrm{mmol} / 1(13.4 \%)$ respectively during the first 3 weeks of substitution (Table 6). The corresponding changes in the margarine group were $-0.39 \mathrm{mmol} / 1 \quad(6.3 \%)$ and $-0.33 \mathrm{mmol} / 1(7.8 \%)$. Cholesterol levels recovered in both groups between weeks 3 and 6 , but remained below the baseline values until the end of the post-experimental period. The HDL-C:TC ratio (\%) increased especially during rapeseed oil substitution. In the control group the changes in cholesterol levels were minor and not significant.

Rapeseed oil substitution at 3 weeks resulted in a decrease of $9.4 \%(-0.64 \mathrm{mmol} / \mathrm{l})$ in TC and $16.3 \%(-0.79 \mathrm{mmol} / 1)$ in the LDL-C of subjects with baseline TC levels higher than $6.0 \mathrm{mmol} / \mathrm{l}$. In the margarine group the corresponding reductions were $6.8 \%$ $(-0.45 \mathrm{mmol} / 1)$ and $7.4 \%(-0.34 \mathrm{mmol} / \mathrm{l})$. An increase in serum TG levels was observed during the experimental period in the rapeseed-oil and control groups $(P<0.05$; Table 6$)$.

\section{Relationships between plasma fatty acid composition and serum cholesterol}

The relationships between plasma fatty acids and serum cholesterol were determined by regression analysis. Due to the large number of tests performed the significance level was set at $P<0.01$.

Of the plasma phospholipids, $\alpha$-LLA was the only fatty acid which correlated significantly with the LDL-C level before substitution in the combined rapeseed-oil and margarine group $(r-0 \cdot 40, P<0 \cdot 01, n 43)$.

The increase in the linoleic: stearic acid ratio in plasma phospholipids correlated with the 


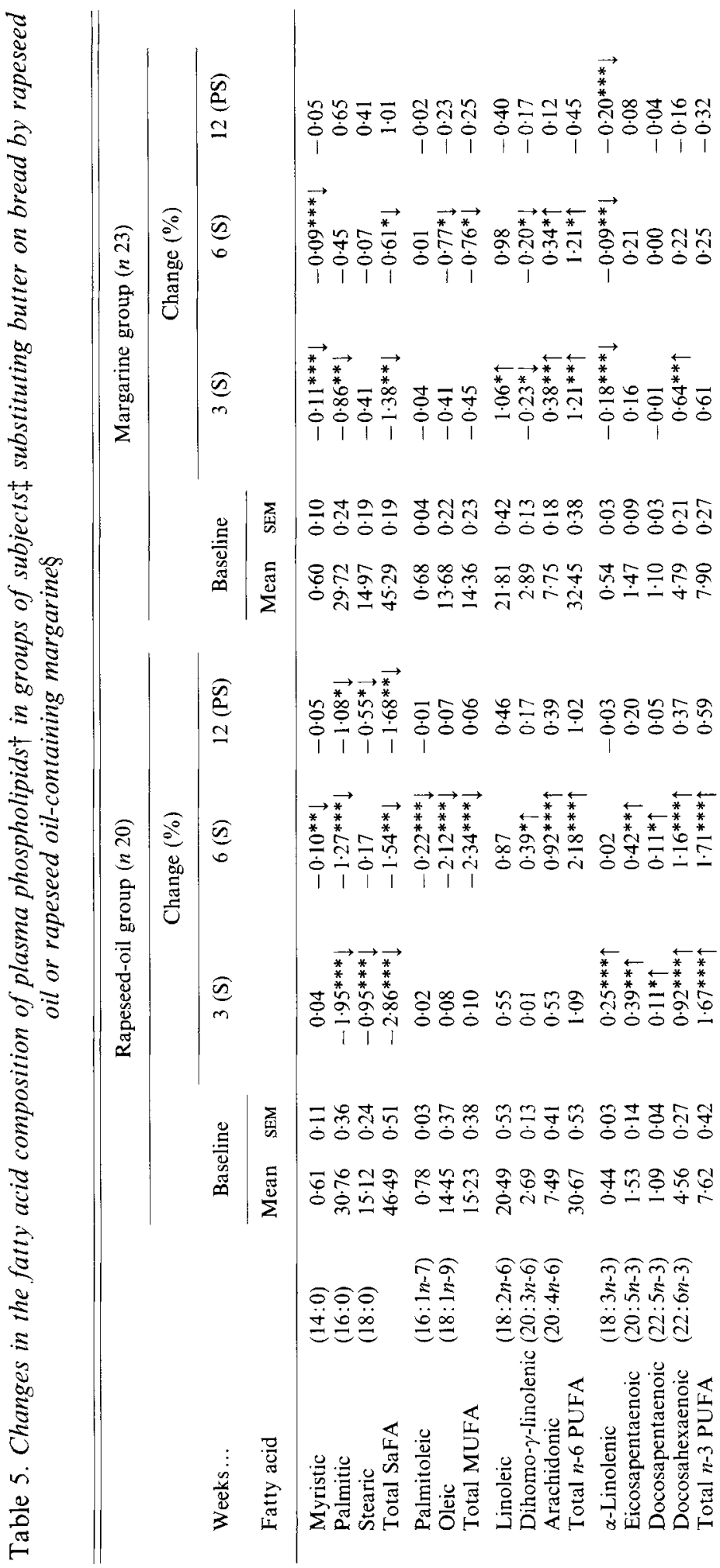




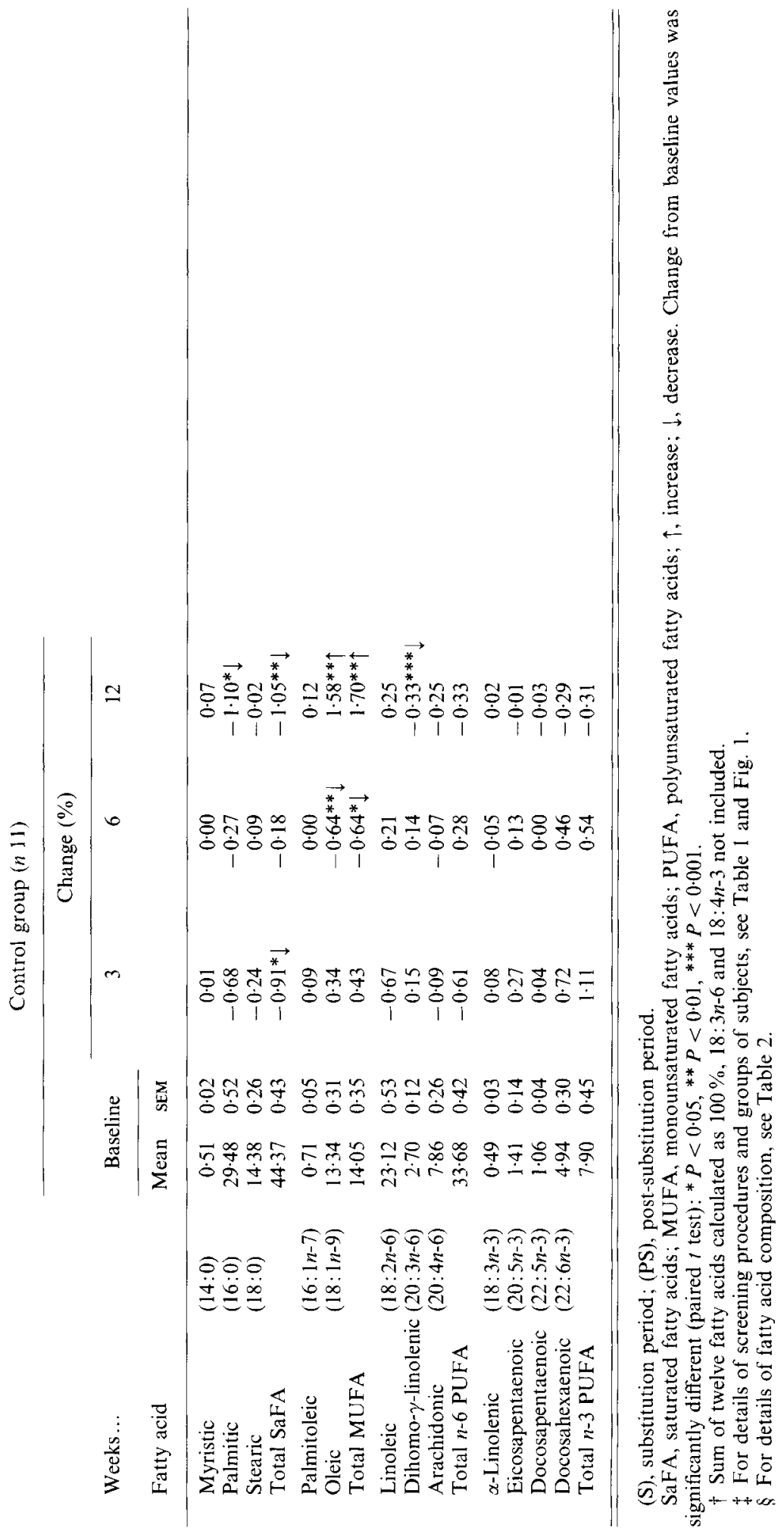




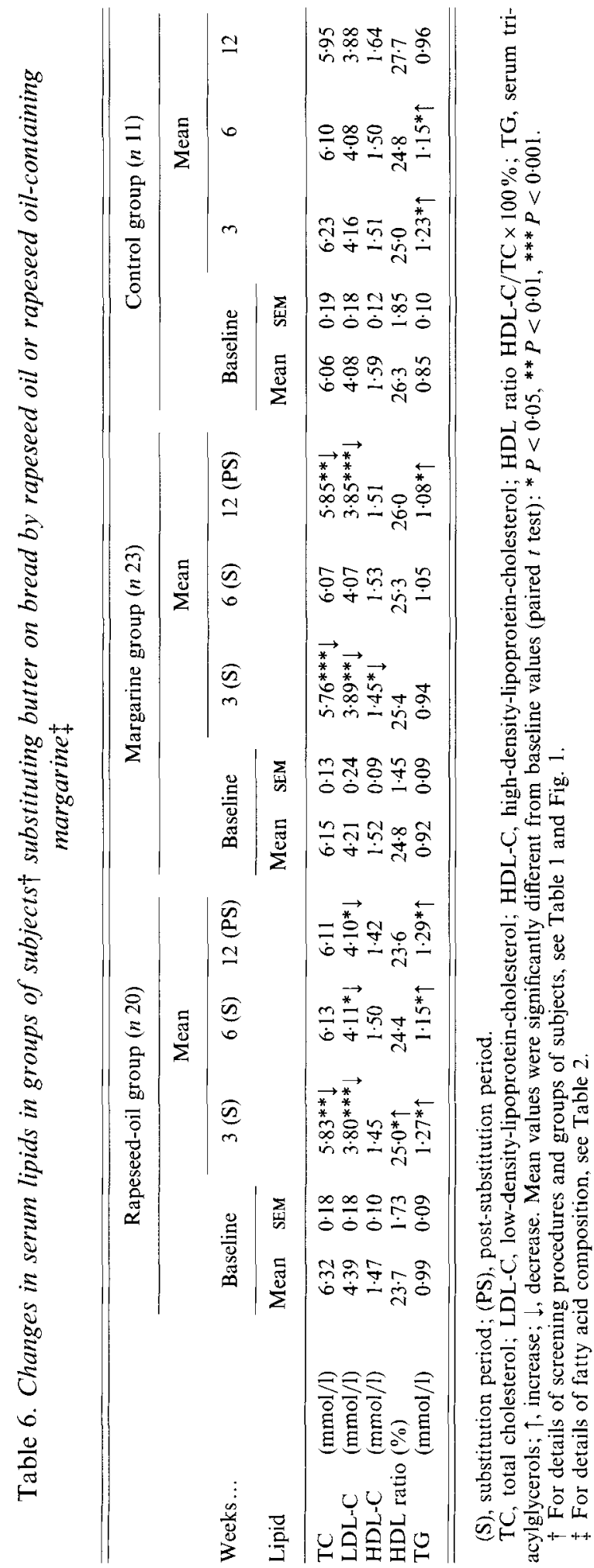


Rapeseed oil group

(a)

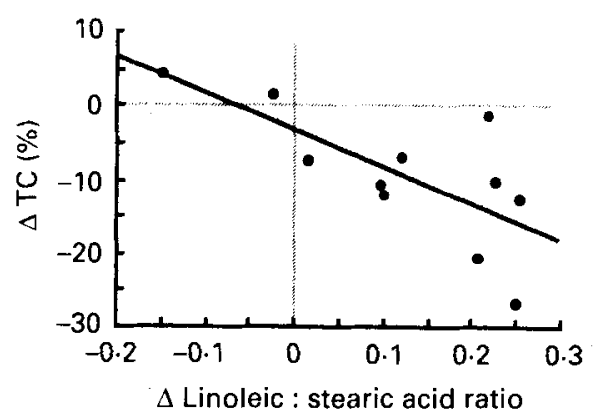

(c)

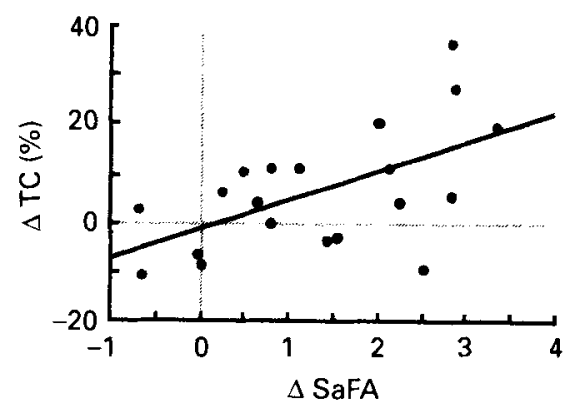

Margarine group

(b)

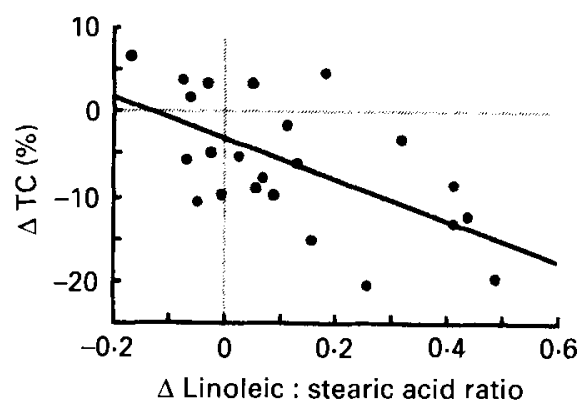

$(d)$

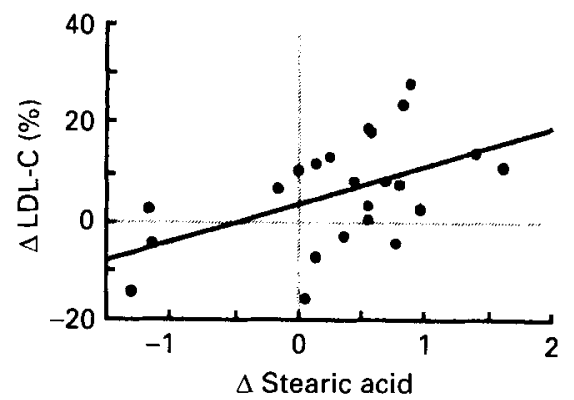

Fig. 2. Relationships between changes in plasma phospholipid fatty acids $(\triangle ; \%)$ and fatty acid ratios and serum cholesterol during substitution of butter on bread by rapeseed oil or rapeseed oil-containing margarine. $(a, b)$ Weeks 1-3, $(c, d)$ weeks 4-6. TC, total cholesterol; SaFA, saturated fatty acids; LDL-C, low-density-lipoproteincholesterol. (a) $r-0.71, P=0.01, n 11 ;(b) r-0.59, P<0.01, n 23 ;(c) r+0.58, P<0.01, n 20 ;(d) r+0.51$, $P=0.01, n 23$. For details of butter substitutes and procedures, see pp. 640-642, Tables 1 and 2 and Fig. 1.

decrease in TC $(r-0 \cdot 71, P=0 \cdot 01)$ in the rapeseed-oil group during the first 3 weeks among subjects with TC baseline values higher than $6.0 \mathrm{mmol} / 1(n \mathrm{ll})$ (Fig. 2(a)). The increase in this ratio was $9 \cdot 2 \%$ and the decrease in TC $9.4 \%$. Slight correlation existed between the rise in the proportion of $\alpha$-LLA and the fall in TC $(r-0.62, P<0 \cdot 05)$.

The increase in the linoleic: stearic acid ratio in plasma phospholipids (Fig. 2(b)) was significantly correlated with the reduction in TC in the margarine group $(r-0.59, P<$ 0.01 ). The change in the linoleic: stearic acid ratio corresponded to an increase of $8.1 \%$, whereas the decrease in TC was $6.0 \%$. In this aspect, the substitution groups did not differ significantly from each other. The change in the proportion of stearic acid at the total plasma level correlated positively with that of LDL-C in the margarine group $(r+0 \cdot 53$, $P<0 \cdot 01$ ).

The cholesterol values increased in both substitution groups between weeks 3 and 6 . In the rapeseed-oil group the change in SaFA in plasma phospholipids (Fig. 2(c)) correlated significantly with that of TC $(r+0.58, P<0.01, n 20)$. During the same period the increase in LDL-C (Fig. $2(d)$ ) correlated with that of stearic acid in the margarine group $(r+0 \cdot 51$, $P=0 \cdot 01)$. 


\section{DISCUSSION}

Dietary studies are often initiated with a typical western diet characterized by a high proportion of saturated fat (Mensink \& Katan, 1987; McDonald et al. 1989). In the present study the effects of a partial, easily implemented rapeseed oil and margarine substitution on serum lipids were monitored. Rapeseed oil was used on bread as a water-oil emulsion. The fatty acid composition of the emulsion was similar to that of zero-erucic acid rapeseed oil (Table 2). Since no other changes were made in the subjects' habitual diet, the fat intake varied greatly. Based on the consumption of substitute fats given in portions, rapeseed oil or margarine replaced an average of $23 \%(8-57 \%)$ of the total dietary fat, while fat accounted for roughly $40 \%$ of the total energy intake. This is about the Finnish average. The control group was asked not to make any dietary changes during the study.

\section{Compliance to the substitution}

Due to the marked differences in dietary habits, it was necessary to control the substitution by means of total plasma fatty acid analyses. The fatty acid composition of plasma mainly resembles that of the TG reflecting the type of dietary fat consumed before blood sampling (Dougherty et al. 1987; Moilanen, 1987).

The relative amount of SaFA in total plasma reached its lowest value at 6 weeks (Table 4 ), reflecting a reduced intake of butter during substitution. The use of rapeseed oil was clearly verified by the significant rise in the $\alpha$-LLA content, and this correlated significantly with the dose. The proportion of LA was increased in both substitution groups, and the maximum levels were reached at 6 weeks (Table 4). In contrast to the data obtained by dietary diaries, rapeseed oil did not cause any considerable increase in plasma OA level, although $\mathrm{OA}$ was the main compound in the substitute fat. The rise in serum TG concentration during rapeseed oil substitution was statistically significant, but it was minor and might be due to the seasonal variation, because a similar change occurred in the control group.

The analyses also showed that there was a lower proportion of SaFA and higher proportion of LA in total plasma in all the groups at the end of May (12 weeks) than in the beginning of February (baseline). These differences, as well as those in serum cholesterol levels (Table 6), are most probably due to seasonal fluctuation. It is known that TC levels are lower and HDL-C levels higher during summer (Thompson, 1989).

\section{Changes in the fatty acid composition of plasma phospholipids}

The fatty acid composition of plasma phospholipids is less variable than that of total plasma, and reflects the type of dietary fat consumed over a period of weeks or even months (Dougherty et al. 1987; Moilanen, 1987). If the changes are expected to be small then a highly reproducible analytical technique is required. The GLC data indicated that the variation of major SaFA such as palmitic and stearic acids in plasma phospholipids was very low. Despite their high resolution and reproducibility in GLC analysis (RSD $2 \cdot 1 \%$ ), the methodological variation represented 18 and $9 \%$ of their total variation respectively, in the phospholipids of 100 subjects. The corresponding 'error' variation for LA and OA, the two other major compounds, was $1 \%$ only (Seppänen-Laakso et al. 1990).

The control group consisted of persons who used both butter and margarine, which would explain the lower SaFA and higher LA levels at the baseline (Tables 4 and 5). However, changes in the fatty acid composition of total plasma and plasma phospholipids were clearly more pronounced in the substitution groups.

During rapeseed oil substitution the major decrease in the proportion of phospholipid SaFA occurred during the first 3 weeks (Table 5), with a simultaneous increase of $n-3$ 
PUFA. The fall in SaFA (2.9\%) was reflected as a drop of only $1.2 \%$ in total plasma (Table 4). Clear differences were also found in the level of $n-3$ PUFA, and the proportion of docosahexaenoic acid (DHA) in total plasma even tended to decrease in all the groups. The increase in phospholipid-DHA during rapeseed oil substitution was probably not high enough to be observed at the total plasma level. Also the decrease in DHA in other lipid fractions, for example in cholesteryl esters, may also be reflected in the fatty acid composition of total plasma.

In contrast to total plasma, the rise in the $\alpha$-LLA content was only temporary, and the decrease at 6 weeks probably indicates its further utilization as the precursor for longerchain $n$-3 PUFA. The proportions of the other $n-3$ PUFA, including EPA, remained at a higher level throughout the substitution period. These results are consistent with earlier studies which have shown that $\alpha$-LLA in rapeseed oil is responsible for the increase in $n$ 3 PUFA (Lassere \& Jacotot, 1983; Renaud et al. 1986), especially EPA, which in turn is a precursor for anti-thrombotic eicosanoids (Crawford, 1983; Renaud et al. 1986; McDonald et al. 1989). It is also evident that the reduction of SaFA further promotes the metabolism of $\alpha$-LLA, since SaFA are known to suppress the activity of the elongating and desaturating enzymes (Crawford, 1983; Holman, 1986). It should be noted that the average intake of $\alpha$-LLA derived from rapeseed oil supplementation represented only $0.8 \%$ of total energy, which corresponds to the minimum dietary requirement for this compound (0.5-1\%) (Holman, 1986; Jacotot et al. 1986). The most marked rise in $n-6$ PUFA in the rapeseed-oil group did not occur until after 6 weeks, and was associated with a simultaneous decrease in MUFA (Table 5).

The three families of unsaturated fatty acids are competitive and an increase in the proportion of dietary $\alpha$-LLA $(n-3)$, LA $(n-6)$ or OA (n-9) suppresses the metabolic products derived from the other two. The relative competitive effect is $\alpha$-LLA $>$ LA $>$ OA (Holman, 1986). Considerable amounts of these fatty acids are present in rapeseed oil. The changes in the proportions of the corresponding fatty acid families in plasma phospholipids occurred in accordance with this competitive ranking in the rapeseed oil group; an increase in $n-3$ PUFA at 3 weeks, then an increase in $n-6$ PUFA at 6 weeks at the expense of MUFA (n-9) (Table 5). These effects cannot be seen as clearly among total plasma fatty acids (Table 4). The competitive action of essential fatty acids during the 6 week substitution period would also explain why the proportion of MUFA was not prominent.

In the margarine group, the changes in total plasma fatty acids resembled those in the rapeseed-oil group, but in phospholipids they were usually minor (Table 5). The most prominent change in plasma phospholipids occurred from SaFA to $n-6$ PUFA already during the first 3 weeks of substitution. This could be expected, since no competition existed with $n-3$ PUFA owing to the minor proportion of $\alpha$-LLA in margarine. At 6 weeks the proportion of $n-6$ PUFA remained at a higher level and suppressed the MUFA, as was the case in the rapeseed-oil group.

The present results show that the two dietary essential fatty acids, $\alpha$-LLA and LA, are immediately utilized for further metabolism when even small amounts of butter are replaced by rapeseed oil. Thus, rapeseed oil, although having moderate amounts of these compounds only, can be a primary source of essential fatty acids rather than that of monoenes in the diet of butter users.

\section{Serum cholesterol v. fatty acid compositions}

When the correlations between serum cholesterol and plasma fatty acid compositions were studied, LA, $\alpha$-LLA and stearic acids were the acids that most often gave significant associations. This was not only the case before dietary supplementation, but also when the changes in TC and LDL-C were analysed (Fig. 2). 
In addition to the increasing effect on $n-3$ PUFA, $\alpha$-LLA when incorporated into plasma phospholipids, may also have an important function with cholesterol, which is supported by the negative correlation with the rapeseed-oil diet. Further evidence is the inverse relationship with LDL-C levels in the combined group before substitution $(P<0.01, n 43)$.

The close relationship found between stearic acid and cholesterol may be confusing, since it has been suggested that dietary stearic acid does not raise the plasma cholesterol concentration (Bonanome \& Grundy, 1988). However, in adipose tissue, which reflects the long-term dietary fat intake, considerable differences in the level of SaFA and PUFA have been reported. It is of special interest that the proportion of stearic acid is twice as high (about $7 \%$ ) in Finnish and Scottish populations compared with that in Italy $(3.6 \%)$ (Riemersma et al. 1986). The corresponding percentages of linoleic acid were 8 and 13.5, thus indicating an approximate linoleic:stearic acid ratio of 1.1 and 3.8 for Finnish and Italian populations respectively. This supports the prominent role of PUFA and stearic acid observed in the present study. Despite the fact that there was a clear decrease in the proportion of total SaFA and palmitic acid in plasma phospholipids during the first 3 weeks of substitution (Table 5), they did not correlate with that of TC or LDL-C. The closer relationships with stearic acid than with palmitic acid may simply arise from the high intake of stearic acid in the subjects' habitual diet.

The relationships between stearic acid and serum cholesterol may be obscured not only by the highly variable proportions of stearic acid in the diet but also by endogenous synthesis of stearic acid from palmitic acid (Sprecher, 1983). A continuous decrease in total plasma SaFA including stearic acid, and probably also in dietary cholesterol (Table 3), appeared at 6 weeks, reflecting the further reduction of butter in the diet. Despite this, the relative amount of stearic acid in plasma phospholipids simultaneously increased to the baseline level (Table 5). An increase in the conversion of palmitic acid to stearic acid is one possible explanation for this restoration phenomenon. The partial increase in cholesterol values at 6 weeks (Table 6) may also be of endogenous origin. In this case, the increase in stearic acid and also in total SaFA were correlated with the change in serum cholesterol (Fig. 2(c,d)).

The reduction of $9.1 \%$ in LDL-C levels without lowering the HDL-C values during 6 weeks of rapeseed oil substitution is significant when we consider the low degree of substitution. Although the increase in dietary MUFA was not reflected at the plasma fatty acid levels, the effect of MUFA may be associated with HDL-C, which is known to remain unchanged during high-MUFA diet (Mensink \& Katan, 1987; Grundy, 1987).

The results show that even minor rapeseed oil substitution brings about favourable effects on the serum lipid profile in butter users. The present study also demonstrates close relationships between serum cholesterol and plasma phospholipid-LA, $\alpha$-LLA and stearic acids. The decrease in SaFA and increase in both $n-3$ and $n-6$ PUFA in plasma phospholipids are the most desirable changes at the fatty acid level. Furthermore, the decrease in TC and LDL $-C$ values without a reduction of HDL-C are factors that can be expected to lower the risk of coronary heart disease at the population level.

\section{REFERENCES}

Ackman, R. G. (1983). Chemical composition of rapeseed oil. In High and Low Erucic Acid Rapeseed Oils, pp. 85-129 [F. D. Sauer and J. K. G. Kramer, editors]. Toronto, New York and London: Academic Press.

Bonanome, A. \& Grundy, S. M. (1988). Effect of dietary stearic acid on plasma cholesterol and lipoprotein levels. New England Journal of Medicine 318, 1244-1248.

Crawford, M. A. (1983). Background to essential fatty acids and their prostanoid derivatives. British Medical Bulletin 39, 210-213.

Dougherty, R. M., Galli, C., Ferro-Luzzi, A. \& Iacono, J. M. (1987). Lipid and phospholipid fatty acid 
composition of plasma, red blood cells, and platelets and how they are affected by dietary lipids: a study of normal subjects from Italy, Finland, and the USA. American Journal of Clinical Nutrition 45, 443-455.

Friedewald, W. T., Levy, R. I. \& Fredrickson, D. S. (1972). Estimation of low density lipoprotein cholesterol in plasma without use of the preparative ultracentrifuge. Clinical Chemistry 18, 499-502.

Grundy, S. M. (1987). Monounsaturated fatty acids, plasma cholesterol, and coronary heart disease. American Journal of Clinical Nutrition 45, 1168-1175.

Hegstedt, D. M., McGandy, R. B., Myers, M. L. \& Stare, F. J. (1965). Quantitative effects of dietary fat on serum cholesterol in man. American Journal of Clinical Nutrition 17, 281-295.

Holman, R. T. (1986). Nutritional and biochemical evidences of acyl interaction with respect to essential polyunsaturated fatty acids. Progress in Lipid Research 25, 29-39.

Jacotot, B., Lassere, M. \& Mendy, F. (1986). Effects of different diets rich in polyunsaturated fatty acids on plasma phospholipids in the human. Progress in Lipid Research 25, 185-188.

Keys, A. (1970). Coronary heart disease in seven countries. Circulation 41, Suppl. 1, 1-211.

Laakso, I. (1986). An analytical and breeding study on fatty acids in summer turnip rape (Brassica campestris $\mathbf{L}$. var. annua). Journal of Agricultural Science in Finland 58, 103-141.

Lassere, M. \& Jacotot, B. (1983). Effets a long terme de plusieurs graisses alimentaires (dont l'huile de colza) sur les lipides seriques d'une population de religieuses benedictines (Long-term effects of several dietary fats (including rapeseed oil) on serum lipids in a Benedictine population). Proceedings of the 6th International Rapeseed Conference, Paris, France, pp. 1653-1659. Paris: GCIRC.

Logan, R. L., Thomson, M., Riemersma, R. A., Oliver, M. F., Olsson, A. G., Rössner, S., Callmer, E., Walldius, G., Kaijser, L., Carlson, L. A., Lockerbie, L. \& Lutz, W. (1978). Risk factors for ischaemic heart-disease in normal men aged 40. Edinburgh-Stockholm study. Lancet $\mathbf{i}, 949-954$.

McDonald, B. E., Gerrard, J. M., Bruce, V. M. \& Corner, E. J. (1989). Comparison of the effect of canola oil and sunflower oil on plasma lipids and lipoproteins and on in vivo thromboxane $\mathrm{A}_{2}$ and prostacyclin production in healthy young men. American Journal of Clinical Nutrition 50, 1382-1388.

Mensink, R. P. \& Katan, M. B. (1987). Effect of monounsaturated fatty acids versus complex carbohydrates on high-density lipoproteins in healthy men and women. Lancet i, 122-124.

Miettinen, T., Naukkarinen, V., Huttunen, J. K., Mattila, S. \& Kumlin, T. (1982). Fatty-acid composition of serum lipids predicts myocardial infaretion. British Medical Journal 285, 993-996.

Moilanen, T. (1987). Short-term biological reproducibility of serum fatty acid composition in children. Lipids 22 , 250-252.

Nestel, P. J. (1987). Polyunsaturated fatty acids (n-3,n-6). American Journal of Clinical Nutrition 45, $1161-1167$.

Renaud, S., Godsey, F., Dumont, E., Thevenon, C., Ortchanian, E. \& Martin, J. L. (1986). Influence of long-term diet modification on platelet function and composition in Moselle farmers. American Journal of Clinical Nutrition 43, 136-150.

Riemersma, R. A., Wood, D. A., Butler, S., Elton, R. A., Oliver, M., Salo, M., Nikkari, T., Vartiainen, E., Puska, P., Gey, F., Rubba, P., Mancini, M. \& Fidanza, F. (1986). Linoleic acid content in adipose tissue and coronary heart disease. British Medical Journal 292, 1423-1427.

Sanders, T. A. B. \& Younger, K. M. (1981). The effect of dietary supplements of $\omega 3$ polyunsaturated fatty acids on the fatty acid composition of platelets and plasma choline phosphoglycerides. British Journal of Nutrition 45, 613-616.

Savoie, L., Navratil, O., Moorjani, S. \& Lupien, P. J. (1983). Effets comparatifs de l'huile de tournesol et de l'huile de colza a faible teneurs en acide erucic dans le traitement de l'hypercholesterolemie familiale heterozygote (Comparative effects of sunflower oil and low erucic acid-rapeseed oil in the treatment of heterozygous familial hypercholesterolaemia). Proceedings of the 6th International Rapeseed Conference, Paris, France, pp. 1700-1705. Paris: GCIRC.

Seppänen, R. (1987). NUTRICA - A computerized food table based programme for estimation of nutrient intake (in Finnish). Kotitalous 3, 20-22.

Seppänen-Laakso, T., Laakso, I. \& Hiltunen, R. (1990). Simultaneous analysis of bound and free fatty acids in human plasma by quantitative gas chromatography. Acta Pharmaceutica Fennica 99, $109-117$.

Sprecher, H. (1983). The mechanisms of fatty acid chain elongation and desaturation in animals. In High and Low Erucic Acid Rapeseed Oils, pp. 385-411 [F. D. Sauer and J. K. G. Kramer, editors]. Toronto, New York and London: Academic Press.

Thompson, G. R. (1989). A Handbook of Hyperlipidaemia, pp. 1-21 [G. R. Thompson, editor]. London: Current Science Ltd.

Viikari, J. (1976). Precipitation of plasma lipoproteins by PEG-6000 and its evaluation with electrophoresis and ultracentrifugation. Scandinavian Journal of Clinical and Laboratory Investigation 36, 265-268.

Weaver, B. J., Corner, E. J., Bruce, V. M., McDonald, B. E. \& Holub, B. J. (1990). Dietary canola oil: effect on the accumulation of eicosapentaenoic acid in the alkenylacyl fraction of human platelet ethanolamine phosphoglyceride. American Journal of Clinical Nutrition 51, 594-598. 